\title{
Ultrasonic piezotome surgery: is it a benefit for our patients and does it extend surgery time? A retrospective comparative study on the removal of 100 impacted mandibular 3rd molars
}

\author{
Angelo Troedhan ${ }^{1}$, Andreas Kurrek ${ }^{2}$, Marcel Wainwright ${ }^{3}$ \\ ${ }^{1}$ Center for Facial Esthetics, Vienna, Austria; \\ ${ }^{2}$ Implantology Clinic Ratingen, Ratingen, Germany; \\ ${ }^{3}$ Implantology Clinic Kaiserswerth, Dusseldorf, Germany. \\ Email: troed@aon.at
}

Received 28 October 2011; revised 3 November 2011; accepted 9 December 2011.

\section{ABSTRACT}

Aim of the study was to evaluate if there is a constant and significant reduction in traumaticity when massively traumatic oral surgical procedures such as the removal of third molars are conducted with only ultrasonic surgical devices (Piezotomes) expressed in a reduction of postsurgical pain and swelling on the patient's side since such clinical experiences by the authors suggested this. Since oral surgeons criticize a higher time consumption for surgeries with Piezotomes also the objective time consumption was evaluated and compared to the traditional methods. Material and Methods: 56 female and male patients were selected that already underwent a removal of an impacted third mandibular molar on one side with rotary instruments by bone destructive burring with a still persisting comparable third mandibular molar on the contralateral side complaining about recurrent pain episodes and were already documented for pain and swelling before. The ultrasonic surgical removal with the Piezotome was conducted with a buccal osteotomy of the compacta lateral to the impacted third molar, preservation of the resected compacta in saline solution, removal of the third molar by single or multiple dentotomy and full anatomical restitution of the surgical site with the preserved buccal compacta. The swelling was documented by kephalometry 24/48/72 hours and 1 week post surgery, the pain index by the total consumption of ibuprofen- $400 \mathrm{mg}-$ tablets. Lesions of the mandible nerve were documented. Netto surgery time was taken from the first incision to the last suture of the procedure. Results: 6 patients had to be excluded from evaluation due to incomplete post surgical follow up. A signifycant (***, $p>0.999$ ) decrease in pain and swelling of $50 \%$ was detected both for the parameters swelling and pain with Piezotome-surgery. No lesions of the mandible nerve were detected with Piezotome surgery whereas surgery with rotary instruments resulted in $16 \%$ hypesthesia at least up to one week. Although netto surgery time was approximately $\mathbf{5 0 \%}$ longer when done with the Piezotome at the beginning the time consumption normalized with the growing experience of the surgeons back to the time schedule when surgery was performed with rotary instruments revealing no significant differences $(-, p<0.73)$. Conclusions: The results of this retrospective study suggest that Piezotome-surgery is superior in atraumaticity and soft-tissue safety compared to traditional procedures with burs and grants the patients significantly less post surgical pain and swelling. Although-as it is with all new surgical tools and protocols-surgery time is longer at the beginning when purely working with ultrasonic surgical devices time consumption reduces to normal values after a learning curve.

Keywords: Ultrasonic Surgery; Piezotome; Rotating Instruments; Post Surgical Swelling; Post Surgical Pain; Impacted Mandibular Third Molars; Osteotomy

\section{INTRODUCTION}

In the year 2004 a new surgical device was introduced to oral and craniomaxillofacial surgery developed by Prof. Tomaso Vercellotti derived from ultrasonic scalers used in the everyday dental office. The "Piezosurgery"-device (Mectron) had more oscillating force thus enabling the surgeon to effectively work on bone and dentin [1].

Like ultrasonic scalers ultrasonic surgery devices oscillate between an average of 24 to $36 \mathrm{KHz}$ and have an energy output at the tip of 15 - 60 Watts.

In the year 2005 Satelec-ACTEON presented its "Pie- 
zotome” with enhanced oscillating behaviour such as a responsive force-modulation and bi-directional force movement of the working tip promising enhanced working speed.

Various histological studies proved ultrasonic surgery to be less traumatic to bone than conventional surgery with rotating instruments thus enhancing and speeding up the healing processes [2-4].

Basic working-tip-sets are common to all ultrasonic surgery devices on the market: a tip-set for osteotomies (micro-scissels, micro-saws), bone collection (rounded and flat bone scrapers), diamond coated balls (1 - $2 \mathrm{~mm}$ ), a sinus-lift-set for lateral sinus-floor-elevations (diamond coated bone-cutter, initial membrane elevator- "trumpet”, various non-cutting elevators), scalers for periodontal surgery (sharp edged and diamond coated) and ligament cutters for tooth extraction.

A tip-set for the minimal-invasive hydrodynamic ultrasonic cavitational sinuslift (HUCSL-“INTRALIFT”) was developed by the authors 2007 for Piezotome (Satelec-ACTEON) [5].

Although the advantages of ultrasonic surgery such as uncomparable atraumaticity and precision in surgery, poor bleeding surgical site, lossless bone management [6] and soft tissue preservation [7] as well as stimulating effects in bone healing [8] are widely accepted by oral and craniomaxillofacial surgeons ultrasonic surgery is still critiziced to be time consuming and lacking a benefit for the patient when used in the daily routine oral surgery.

To investigate this critic closer the authors established a study protocol to objectively quantify the benefit of ultrasonic Piezotome surgery for patients in daily routine oral surgery and the average duration of surgical procedures compared to conventional procedures with rotating instruments (burs) since the authors observed a signifycant decrease in pain and swelling after completely switching all oral surgery procedures to ultrasonic surgical devices completely abandoning burs in 2006.

\section{MATERIAL AND METHODS}

56 male and female patients at an age between $21 \mathrm{yr}$ and 52 yr were selected for the retrospective study who already underwent the removal of an impacted third molar and were already documented for pain, swelling and surgery time on behalf of a prior study. Patients were anonymized in the study protocol by assigning a number.

All patients had bilateral comparable mandibular impacted third molars with the crown-root axis parallel or at a maximum of 20 degrees ascending to the occlusal plane of the mandible and regular developed second molars in occlusion (Figures 1-3).

Prior to surgery measure spots were marked with a resorbable tattoo-ink for kephalometry in the skin adjacent the mandibular angle on both sides.

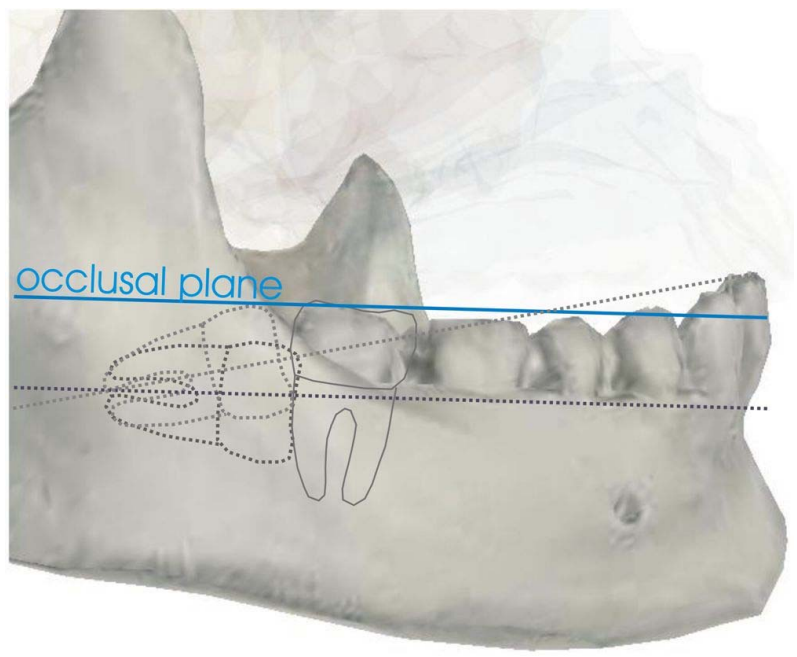

Figure 1. Schematic scheme for eligibility.

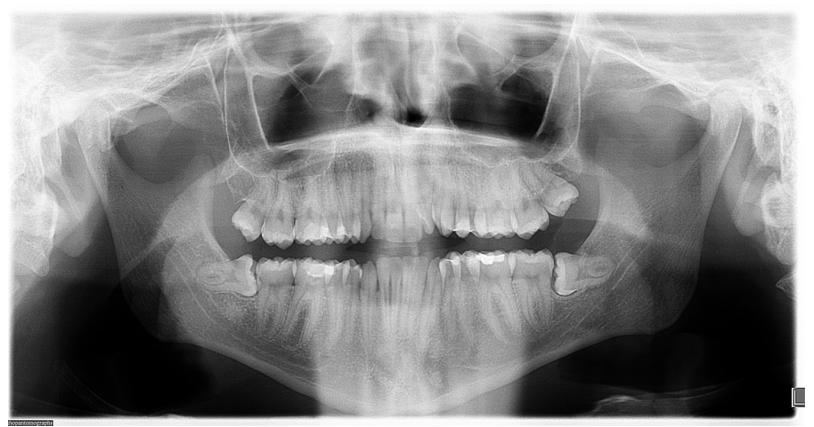

Figure 2. Bilateral horizontally impacted third molars.

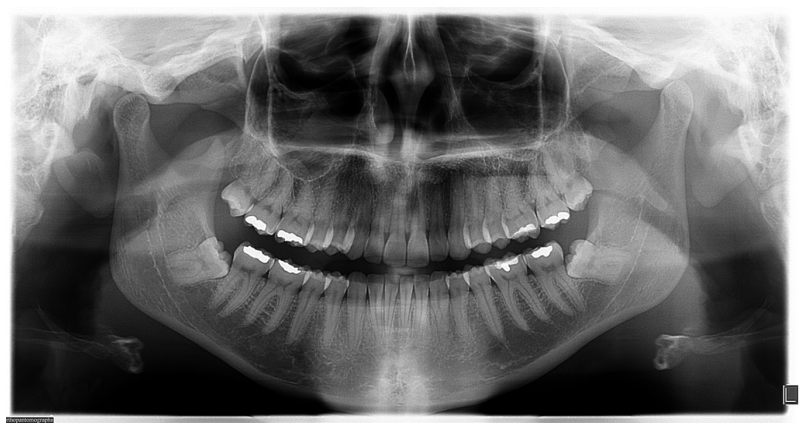

Figure 3. Bilateral angulated impacted third molars.

Before anesthesia a protocolist assitant different from the surgeon measured the skin-distance between the right and left mandibular angle tatoo with a kephalometer and noted it to the study protocol.

All patients already underwent the removal of one impacted third molar on one side under local anesthesia (6 - $8 \mathrm{ml}$ Ultracain-forte) with conventional rotating instruments, periotomes, scissels and leverages (the "traditional” surgical procedure, Figure 4) in the years 20042006 and were already documented by a protocol that was then also followed for the current study. 
With the availability of ultrasonic surgical devices for bone cutting from 2006 the contralateral impacted third molar was removed using the Piezotome (Satelec-ACTEON, Figure 5) and one leverage only under local anesthesia (6 - $8 \mathrm{ml}$ Ultracain forte)

To reduce ambient seasonal influences (ambient temperature, humidity, daylight hours etc.) the ultrasonic surgery was performed $+/-3$ weeks of the date of the prior surgery with rotating instruments.

Both surgical procedures were started with a $45^{\circ}$ mesial-vertical mucoperiostal incision reaching the distal face of the second molar in the mesio-distal line of the crowns central fissure proceeding mesio-distal on the alveolar crest up to the ascending part of the mandible in the retromolar trigonum (Figures $\mathbf{4}$ and $\mathbf{6}$ )

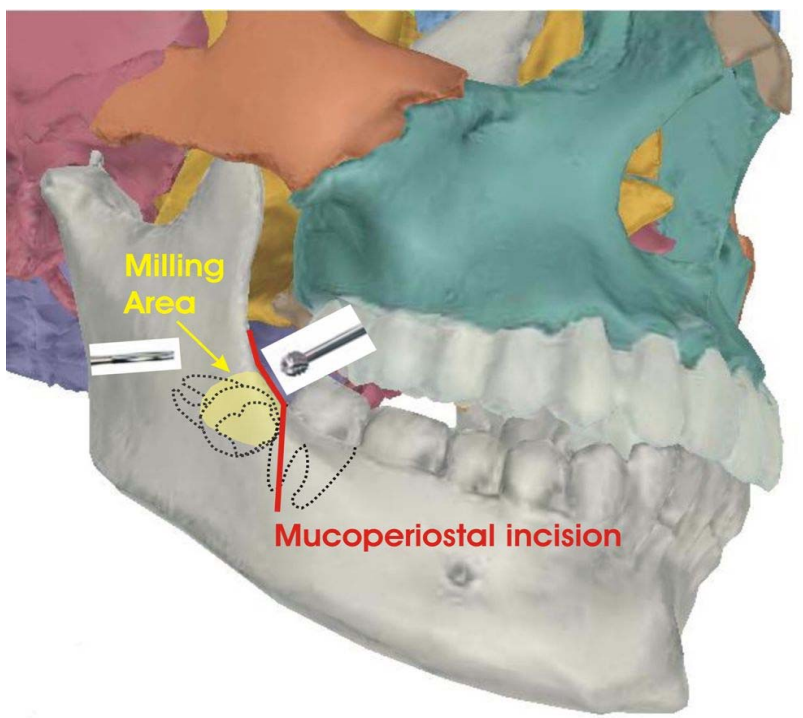

Figure 4. Surgical protocol with rotary instruments: vast removal of buccal compacta bone to reveal the crown, single or multiple dentotomy, removal and wound closure.

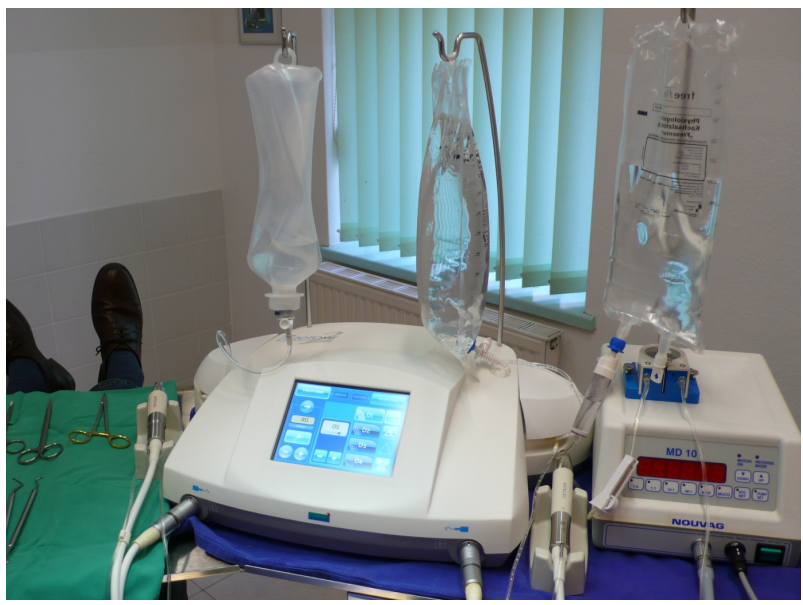

Figure 5. Ultrasonic surgical device piezotome (center; Satelec-ACTEON/France).

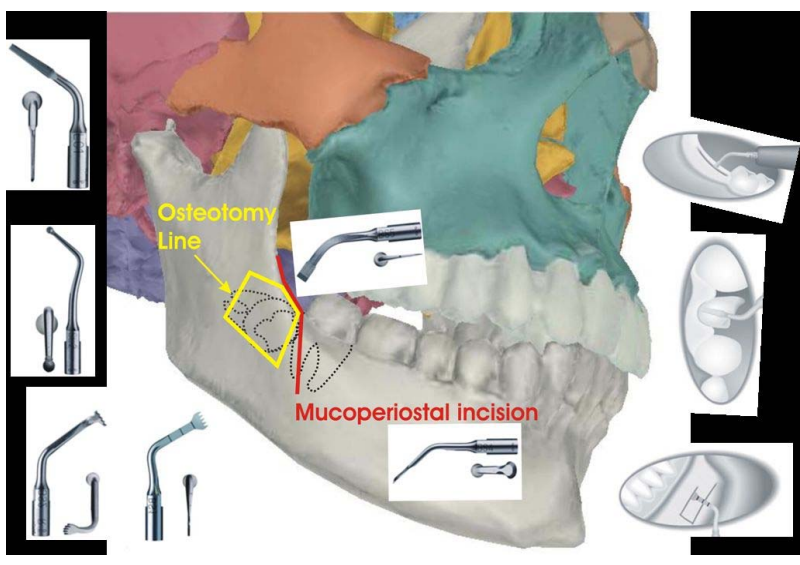

Figure 6. Surgical Protocol with Piezotome: almost lossless osteotomy and luxation of the buccal bone compacta with BS 5 (bone scalpel), cutting of the periodontal ligaments with ligament cutters LC 1-5, dentotomy, removal, reposition of the buccal bone compacta for anatomical correct reconstruction

Conventional surgery (Figure 4) with rotating instruments was performed according to the valid surgical protocol prior to the introduction of ultrasonic Piezotomesurgery: after preparing the mucoperiostal flap with a periostal elevator the alveolar crest and buccal compacta was removed by milling with a 3-mm Tungsten Carbide Bur (Meisinger) until the distal and buccal face of the crown and the first one third of the root could be seen. The horizontally impacted third molar was then cut in two or more pieces in the area of the enamel-dentinemargin with the Tungsten Carbide Bur, the crown or multiple pieces of the crown removed with different leverages, the roots mobilized with the periotome and-if necessary again cut in two separate parts at the furcation and finally removed with leverages.

Ultrasonic surgery (Figure 6) with the Piezotome was performed as follows: the mucoperiostal flap was elevated with the BS4-tip (originally designed for Bone harvesting) and the alveolar crest and buccal compacta resected as one bone block after osteotomy with the ultrasonic scissel BS5 and the micro-bone-saws BS1 and BS2 (Figure 7) thus revealing the distal and entire buccal face of the crown and the buccal sides of the roots in their entire length (Figure 8). The prepared bone block was kept in saline solution for later anatomically correct reconstruction of the surgical site. The crown was separated from the roots by cutting the roots with the diamondcoated ball-tip SL2, then both crown and roots mobilized with the ligament-cutter LC 1 and removed with a leverage (Figure 9). The surgical site was inspected for clinically visible damages of the mandible nerve when applicable (Figure 10). The osteotomed bone block was reponed before wound closure to reconstruct the original anatomy of the surgical site (Figure 11). 


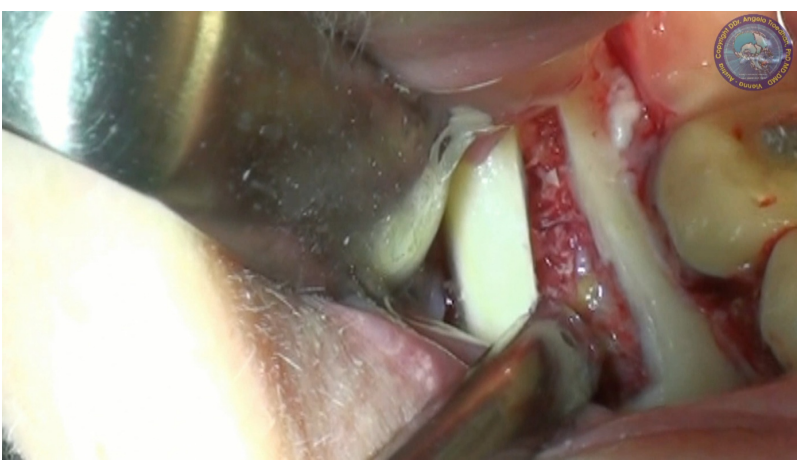

Figure 7. Almost lossless osteotomy and luxation of the buccal compacta.

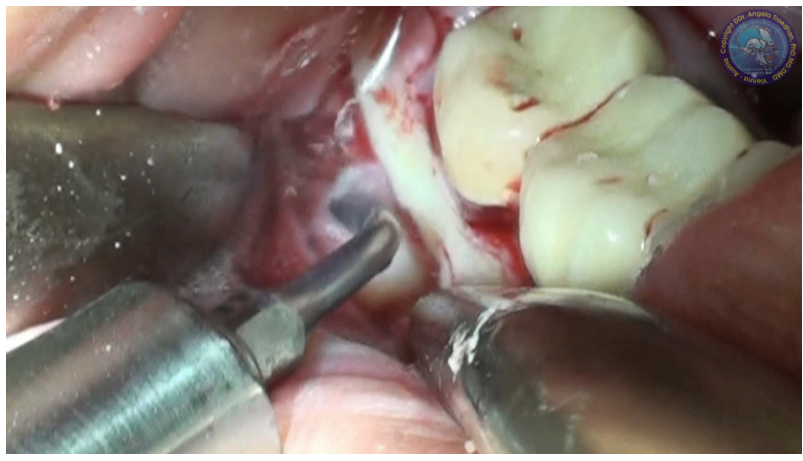

Figure 8. Cutting the periodontal ligament with Ligament Cutters (LC) 1-5 followed by dentotomy.

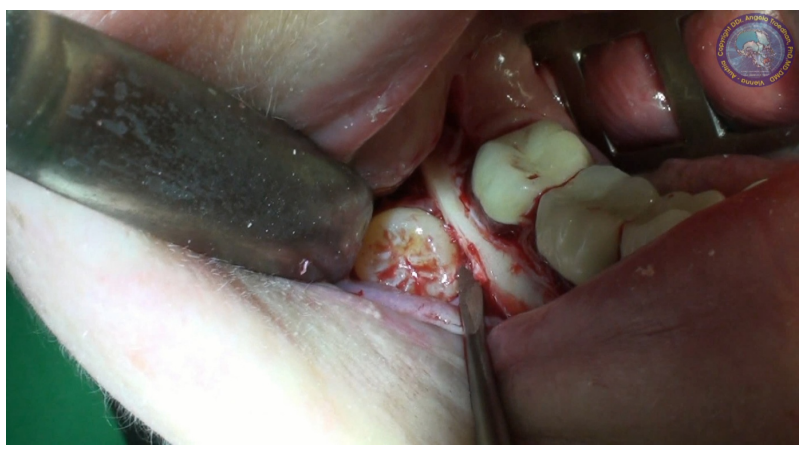

Figure 9. Luxation of the crown followed by removal of the roots.

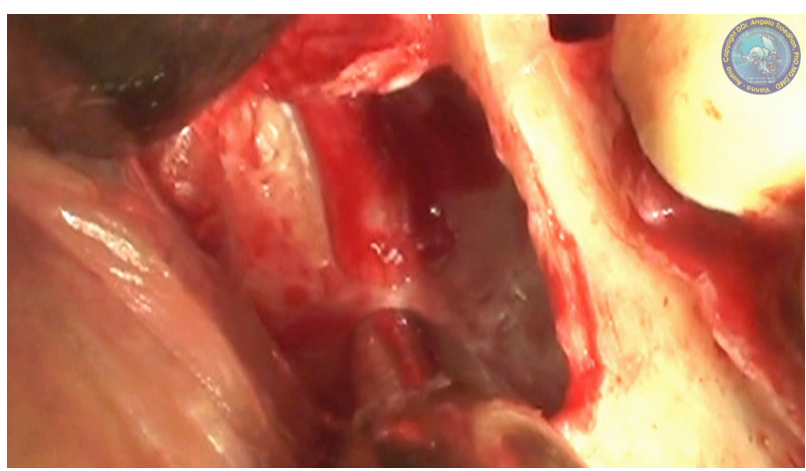

Figure 10. Inspection of the surgical site for lesions of the mandible nerve (if applicable).

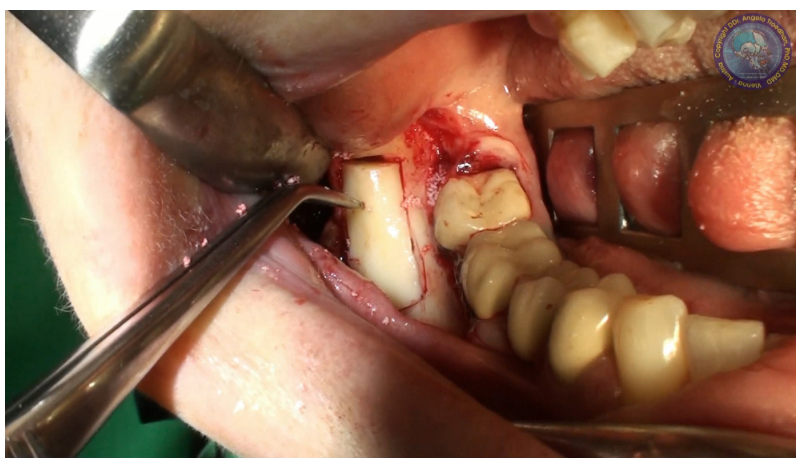

Figure 11. Reposition of the buccal compacta bone plate to reconstruct the original anatomy. No osteosynthesis is needed due to the almost lossless osteotomy cut, the angulated cut design providing "rest surfaces" and the precise refitting.

The time for the surgical procedure was taken from the first mucoperiostal incision to the last suture to close the wound by a standby assistant and noted in the study protocol.

The patients were handed out two blister packs per 10 tablets of the analgetic Dexibuprofen $400 \mathrm{mg}$ ("Seractil forte") and an antibiotic shielding with Clindamycin 300 mg $3 \times 1$ /day 16 capsules after surgery. Unused analgetic tablets had to be brought back and were counted by the protocolist. All patients were advised to apply cool packs and refrain from sport activities and heavy body work in the first 72 hours after surgery.

The skin distance between the tattoo at the angle of the mandible on the non surgery side and the utmost swelling protrusion on the surgery side was measured with a kephalometer by a protocolist assistant different from the surgeon after $24 \mathrm{hr}, 48 \mathrm{hr}, 72 \mathrm{hr}$ and one week $(+/-1 \mathrm{hr})$ and noted to the study protocol. The analgetic tablets brought back by the patients were counted and the usage noted to the study protocol.

Hypesthesia 1 week after surgery in the corresponding lip-half was checked by the two-point discrimination-test and noted - if occurred - to the study protocol.

From 56 patients 50 were included in the study. The causes for the exclusion of 6 patients were: 1 patient did not show up for kephalometry after 24 hours, 2 patients had to be excluded since they failed the 24 hour $+/-1 \mathrm{hr}$ margin for kephalometry by more than 6 hours delay, 1 patient did not show up for kephalometry after $72 \mathrm{hr}$ and 2 patients did not show up for kephalometry after 1 week thus lacking data for objective comparison.

\section{RESULTS}

The average mean value swelling of the patients face undergoing surgery with rotating instruments measured between the utmost lateral protrusion of the cheek on the surgery side and the contralateral tattoo at the angle of the mandible with the kephalometer resulted in a swelling $24 \mathrm{hr}+/-1 \mathrm{hr}$ post surgery of $44 \mathrm{~mm}, 48 \mathrm{hr}+/-1 \mathrm{hr}$ 
$49 \mathrm{~mm}, 72 \mathrm{hr}+/-1 \mathrm{hr} 46 \mathrm{~mm}$ and after one week $5 \mathrm{~mm}$ (Tables 1-4).

The average mean value swelling of the patients face undergoing surgery with the ultrasonic Piezotome-surgical device measured between the utmost lateral protrusion of the cheek on the surgery side and the contralateral tattoo at the angle of the mandible with the kephalometer resulted in a swelling $24 \mathrm{hrs}+/-1 \mathrm{hr}$ post surgery of $20 \mathrm{~mm}, 48 \mathrm{hr}+/-1 \mathrm{hr} 20 \mathrm{~mm}, 72 \mathrm{hrs} \mathrm{+/-} 1 \mathrm{hr} 15$ $\mathrm{mm}$ and after one week $1 \mathrm{~mm}$ (Tables 1-4) which is a significant (***, $\mathrm{p}>0.999$ ) reduction of $50 \%$ compared to the procedure with rotary instruments.

Figure 12 shows the cumulated results of the swelling kephalometry.

The mean value of the patients analgetic intake (Dexibuprofen $400 \mathrm{mg}$ ) was 17 tablets in the group undergoing surgery with rotating instruments (Table 5) and 8 tablets in the group undergoing ultrasonic surgery (Table 5 ) which is a significant (***, p $>0.999)$ reduction of 50 $\%$ of the patient's need for analgetics in the ultrasonic Piezotome-surgery group.
In both groups no full anesthesia of the corresponding mandible nerve was observed 1 week after surgery (0\%).

In the rotary-instrument group 8 patients suffered from hypesthesia in the corresponding lip at the check after 1 week (16\%).

In the ultrasonic Piezotome-group no hypesthesia was observed at the check after 1 week $(0 \%)$.

While the time between the initial mucoperiostal incision and wound closure by suture performed with rotating instruments was constantly at an average of 43 minutes (Table 6) surgery with the ultrasonic device initially took a maximum of 72 minutes but constantly lowered to also an average of 47 minutes (Table 6) finally showing no significant $(-, \mathrm{p}<0.73)$ time difference to procedures with rotary instruments. This might be caused by the fact that the surgeons working procedures in ultrasonic surgery undergoes a "learning-curve" since the single surgical steps are different to the conventional technique with rotating instruments. With growing experience the handling of the ultrasonic device Piezotome is constantly optimised.

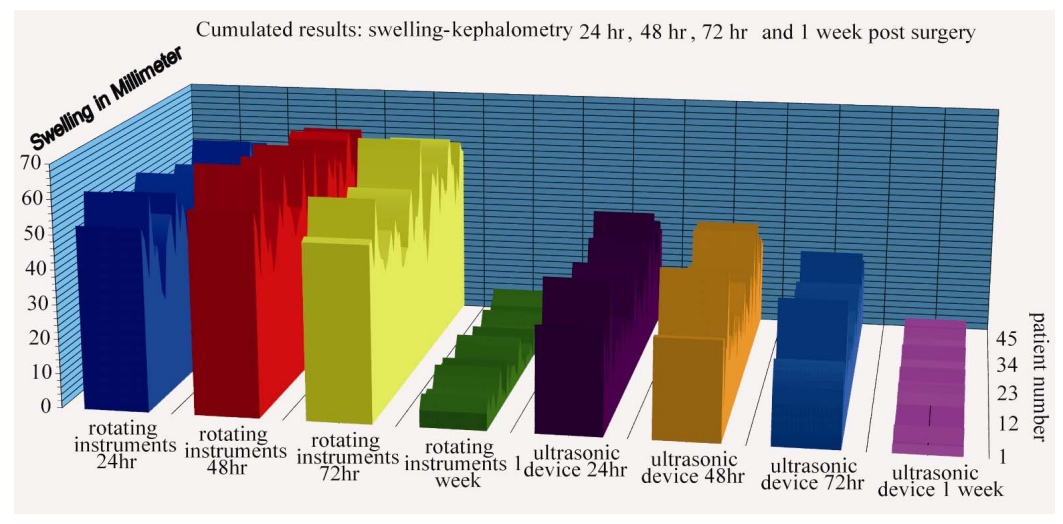

Figure 12. The cumulated results shown in a compiled graph.

Table 1. Comparison of facial edema $24 \mathrm{hr}$ post surgery.

\begin{tabular}{ccc}
\hline Swelling after $24 \mathrm{hr}($ in $\mathrm{mm})$ & Rotary Instruments & Piezotome \\
\hline Mean Value & 44.36 & 20.30 \\
Max & 61 & 41 \\
Min & 28 & 5 \\
Stand. Dev. & 7.3 & 7.99 \\
Significance & $* * *$ & $(\mathrm{p}>0.999)$ \\
\hline
\end{tabular}

Table 2. Comparison of facial edema $48 \mathrm{hr}$ post surgery.

\begin{tabular}{ccc}
\hline Swelling after $48 \mathrm{hr}$ (in mm) & Rotary Instruments & Piezotome \\
\hline Mean Value & 49.38 & 20.54 \\
Max & 72 & 45 \\
Min & 34 & 5 \\
Stand. Dev. & 8.02 & 7.81 \\
Significance & $* * *$ & $(\mathrm{p}>0.999)$ \\
\hline
\end{tabular}

Table 3. Comparison of facial edema $72 \mathrm{hr}$ post surgery.

\begin{tabular}{ccc}
\hline Swelling after $72 \mathrm{hr}$ (in mm) & Rotary Instruments & Piezotome \\
\hline Mean Value & 46.16 & 15.28 \\
Max & 67 & 38 \\
Min & 28 & 3 \\
Stand. Dev. & 7.72 & 6.40 \\
Significance & $* * *$ & $(\mathrm{p}>0.999)$ \\
\hline
\end{tabular}

Table 4. Comparison of facial edema 1 week post surgery

\begin{tabular}{ccc}
\hline Swelling after 1 week (in mm) & Rotary Instruments & Piezotome \\
\hline Mean Value & 4.7 & 1.02 \\
Max & 12 & 5 \\
Min & 0 & 0 \\
Stand. Dev. & 3.22 & 1.61 \\
Significance & $* * *$ & $(\mathrm{p}>0.999)$ \\
\hline
\end{tabular}


Table 5. Comparison of overall analgetic intake (ibuprofen 400 $\mathrm{mg})$.

\begin{tabular}{ccc}
\hline Analgetic intake (tablets) & Rotary Instruments & Piezotome \\
\hline Mean Value & 17.6 & 7.8 \\
Max & 20 & 15 \\
Min & 10 & 1 \\
Stand. Dev. & 3.05 & 3.17 \\
Significance & $* * *$ & $(\mathrm{p}>0.999)$ \\
\hline
\end{tabular}

Table 6. Comparison of duration of surgeries in minutes.

\begin{tabular}{ccc}
\hline Surgery time (in minutes) & Rotary Instruments & Piezotome \\
\hline Mean Value & 42.86 & 46.84 \\
Max & 59 & 72 \\
Min & 28 & 29 \\
Stand. Dev. & 6.69 & 8.48 \\
Significance & - & $(\mathrm{p}<0,73)$ \\
\hline
\end{tabular}

While most of the time is lost with the ultrasonic surgical device Piezotome in cutting the buccal compacta as a precise bone block to reveal the impacted third molar the fast and destructive milling away of the cortical bone with burs to reveal the third molar performs faster but this time is regained by the perfectly visible and nearly blood-free surgical site, the easiness of the separation of the crown from the roots without fear to damage the mandible nerve and the easy luxation of the tooth fragments out of it's bone site with the ultrasonic ligament cutters.

\section{DISCUSSION}

The results of this study suggest that it can be stated as proven that pure ultrasonic surgery procedures with its nearly lossless and ultimate precise bone management can completely replace conventional surgery techniques with hand instruments, rotating instruments, periotomes and most levers and dental pliers to the patient's benefit.

With an average of 50\% less swelling and 50\% post surgical pain reduction and the preservation of critical soft tissues such as the mandible nerve ultrasonic surgery furthermore reduces the need for additional antiinflammatory and swelling-reducing medication and initiates faster and better wound healing.

The immediate anatomically correct reconstruction of the surgery site avoids long term inconveniences primarily for the patient but also for the surgeon lacking the necessity to treat these inconveniences.

Although the positive effects of application of low frequency ultrasonic waves between 25 and $40 \mathrm{KHz}$ on the healing of soft and hard tissue on a histophysiological and histomorphological level were already published in various Scientific Journals the multiple effects of ultrasonic Piezotome surgery with the current and future devices in oral and craniomaxillofacial surgery have still to be researched into deep to identify the biological pro- cesses on the molecular and humoral level that lead to the clinical results of lesser swelling, pain reduction and faster and complication-poor healing.

Ultrasonic Piezotome surgery-after a "learning curve" in handling the device and individually optimise and economize the surgical procedures-does not consume more time than conventional surgical techniques with rotating instruments in the everyday surgical routine such as teeth extractions, apisectomies, removal of impacted teeth, periodontal surgery etc. and the individual surgeon has no need to recalculate his or her time schedule for routine surgical procedures in his/her office.

On the other hand ultrasonic surgery allows almost atraumatic and minimal invasive surgical procedures that were unthinkable with traditional instruments (such as transcrestal hydrodynamic ultrasonic sinuslifting, flapless crest splitting etc.) thus sparing time, costs, pain and complications to both the surgeon and the patient.

\section{REFERENCES}

[1] Vercellotti, T. (2009) Essentials in piezosurgery: Clinical advantages in dentistry. 1st Edition, Quintessence Publishing Co., San Francisco.

[2] Horton, J.E., Tarpley, T.M.Jr. and Wood, L.D. (1975) The healing of surgial defects in alveolar bone produced with ultrasonic instrumentation, chisel and rotary bur. Oral Surgery, Oral Medicine, Oral Pathology, Oral Radiology, and Endodontology, 39, 536-546.

[3] McFall, T.A., Yamane, G.M. and Burnett, G.W. (1961) Comparison of the cutting effect on bone of an ultrasonic cutting device and rotary burs. Journal of Oral Surgery, Anesthesia and Hospital Dental Service, 19, 200-209.

[4] Aro, H., Kallioniemi, H., Aho, A.J. and KellokumpuLehtinen, P. (1981) Ultrasonic device in bone cutting. A histological and scanning electron microscopical study. Acta Orthopaedica Scandinavica, 52, 5-10. doi:10.3109/17453678108991750

[5] Troedhan, A.C., Kurrek, A., Wainwright, M. and Jank, S. (2010) Hydrodynamic ultrasonic sinus floor elevation-An experimental study in sheep. Journal of Oral and Maxillofacial Surgery, 68, 1125-1130. doi:10.1016/j.joms.2009.12.014

[6] Gleizal, A., Béra, J.-C., Lavandier, B. and Béziat, J.-L. (2007) Piezoelectric osteotomy: A new technique for bone surgery-Advantages in craniofacial surgery. Child's Nervous System, 5, 509-513. doi:10.1007/s00381-006-0250-0

[7] Geha, A.H.J, Gleizal, A., Nimeskern, N. and Béziat, J.-L. (2006) Sensitivity of the inferior lip and chin following mandibular bilateral sagittal split osteotomy using piezosurgery. Plastic and Reconstructive Surgery, 118, 15981607. doi:10.1097/01.prs.0000232360.08768.de

[8] Gleizal, A., Li, S.L., Pialat, J.-B. and Béziat, J.-L. (2006) Transcriptional expression of calvarial bone after treatment with low-intensity ultrasound: An in vitro study. Ultrasound in Medicine \& Biology, 32, 1569-1574. doi:10.1016/j.ultrasmedbio.2006.05.014 